\title{
Resonance broadening and tuning of split ring resonators by top-gated epitaxial graphene on SiC substrate
}

Semih Cakmakyapan, Levent Sahin, Francesco Pierini, Wlodek Strupinski, and Ekmel Ozbay

Citation: Appl. Phys. Lett. 103, 181116 (2013);

View online: https://doi.org/10.1063/1.4828499

View Table of Contents: http://aip.scitation.org/toc/apl/103/18

Published by the American Institute of Physics

\section{Articles you may be interested in}

Tunable metamaterials based on split ring resonators and doped graphene

Applied Physics Letters 103, 011102 (2013); 10.1063/1.4812989

Modulation of mid-infrared light using graphene-metal plasmonic antennas

Applied Physics Letters 102, 131108 (2013); 10.1063/1.4800931

Graphene induced spectral tuning of metamaterial absorbers at mid-infrared frequencies Applied Physics Letters 103, 261111 (2013); 10.1063/1.4858459

Absorption enhancement and total absorption in a graphene-waveguide hybrid structure AIP Advances 7, 025101 (2017); 10.1063/1.4975706

Enhancement of near-infrared absorption in graphene with metal gratings Applied Physics Letters 105, 031905 (2014); 10.1063/1.4890624

Ultra-compact optical modulator by graphene induced electro-refraction effect Applied Physics Letters 103, 061116 (2013); 10.1063/1.4818457

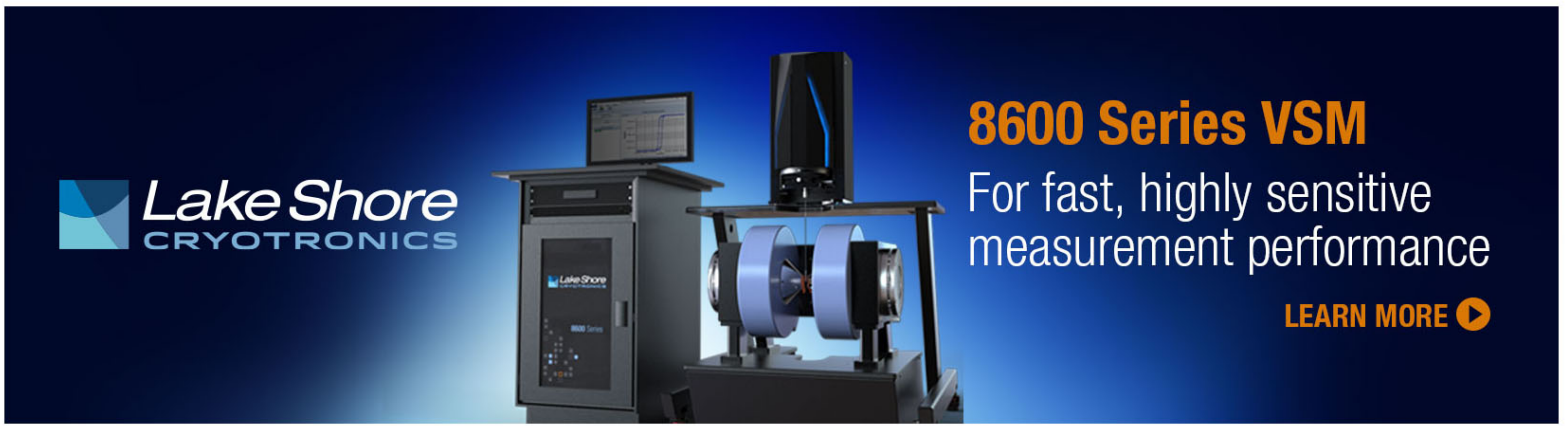




\title{
Resonance broadening and tuning of split ring resonators by top-gated epitaxial graphene on SiC substrate
}

\author{
Semih Cakmakyapan, ${ }^{1,2, a)}$ Levent Sahin, ${ }^{2,3}$ Francesco Pierini, ${ }^{2}$ Wlodek Strupinski, ${ }^{4}$ \\ and Ekmel Ozbay ${ }^{1,2,3}$ \\ ${ }^{1}$ Department of Physics, Bilkent University, 06800 Ankara, Turkey \\ ${ }^{2}$ Nanotechnology Research Center, Bilkent University, 06800 Ankara, Turkey \\ ${ }^{3}$ Department of Electrical and Electronics Engineering, Bilkent University, 06800 Ankara, Turkey \\ ${ }^{4}$ Institute of Electronic Materials Technology, Wolczynska 133, 01-919 Warsaw, Poland
}

(Received 15 August 2013; accepted 16 October 2013; published online 31 October 2013)

\begin{abstract}
Split ring resonators (SRRs) are subwavelength structures that are able to localize and enhance the electromagnetic wave. Controlling the plasmonic resonance behavior of metallic nanostructures, such as SRRs, plays an important role in optoelectronics and nanophotonics applications. Electrically tunable carrier concentration of graphene provides hybrid devices, where the plasmonic structures and graphene are combined. In this paper, we report the design, fabrication, and measurement of a device comprising a SRR array on epitaxial graphene. We obtained resonance broadening and tuning of split ring resonators by utilizing an epitaxial graphene transistor with transparent top-gate. C 2013 AIP Publishing LLC. [http://dx.doi.org/10.1063/1.4828499]
\end{abstract}

Graphene is a monolayer of carbon atoms that are arranged in a two-dimensional honeycomb lattice. This 2D material can be used for wrapping up into fullerenes (0D), for rolling into nanotubes (1D), or for stacking into 3D graphite. ${ }^{1}$ Since graphene has interesting electrical and mechanical properties, it has been an attractive research material in recent years. An electron inside a monolayer graphene sheet has zero effective mass, ${ }^{2}$ and it can travel for micrometers without scattering even at room temperature. ${ }^{3,4}$ Graphene also exhibits many interesting and exotic properties ranging from ballistic electronic transport ${ }^{5-7}$ to the anomalous quantum Hall effect in electrical transport ${ }^{2,7}$ and tunable interband transitions. ${ }^{8,9}$ Graphene has exceptional optical properties. For instance, graphene exhibits saturable absorption constant as a consequence of Pauli blocking. ${ }^{10-12}$ Moreover, non-equilibrium carrier results in hot luminescence. ${ }^{13,14}$ Graphene is a versatile optical material for nanophotonics applications ${ }^{3}$ such as metamaterials ${ }^{15}$ photodetectors,${ }^{16}$ light emitting devices, and ultrafast lasers. ${ }^{17}$ In particular, graphene with its planar nature attracts an increasing research interest as a promising tool for nanoplasmonics and nanoscale electronics. ${ }^{18,19}$ Pioneering works of graphene were focused on the single particle excitation of electrons. On the other hand, the plasmonic properties of graphene layer, which is a result of the collective excitations of 2D massless electrons, has become an emerging research area in recent years. Plasmonic resonances in graphene give outstanding potential for designing optoelectronic devices by its remarkably high absorption. ${ }^{3,20,21}$ Electrical response at terahertz frequencies are high enough to design plasmonic devices. ${ }^{5,22}$ However, the electrical control of plasmon resonance becomes a challenge at optical frequencies as a result of drastically reduced free electron responses with increasing frequency. Graphene, which is a zero bandgap semiconductor, ${ }^{8,9,22}$ is a great candidate to overcome this challenge. Since high frequency interband transition in graphene can be

\footnotetext{
${ }^{\text {a) }}$ Author to whom correspondence should be addressed. Electronic mail: semihc@bilkent.edu.tr
}

exploited through electrical gating, ${ }^{22}$ plasmon resonance of graphene-hybrid device like split ring resonators (SRRs) can be modulated by applying gate voltage, ${ }^{23,24}$ which changes the carrier concentration, thus modulating the optical conductivity. ${ }^{25,26}$ Surface plasmons have the characteristics of concentrating light into a subwavelength volume, which is one of the main tools of plasmonics. ${ }^{1,22}$ Plasmons in graphene is an active research field, ${ }^{20,21,27-29}$ and it is desired to control the plasmon resonances for optical switch applications.

Here, we report the optical response of SRR arrays on epitaxial graphene. Strong localized fields around SRRbased structures couple to the incident field through a resonant process, causing a strong localization of electromagnetic waves. SRRs enhance the incident field and therefore increase the interaction between light and graphene, where the asymmetric broadening and shifting of transmission spectra are studied.

The graphene sample in this study is grown by the sublimation method on $4 \mathrm{H}-\mathrm{SiC}$ polytype. The sample is intercalated by hydrogen, where the temperature of sublimation is $1600^{\circ} \mathrm{C}$, and the reactor pressure is set to $50 \mathrm{mbar}$. The sample is p-type with a carrier concentration of $1.3 \times 10^{13} \mathrm{~cm}^{-2}$, and the mobility is measured to be $3234 \mathrm{~cm}^{2} / \mathrm{Vs}$. In terms of the fabrication of the samples, first of all, a chromium mask is designed and fabricated by electron beam lithography to be further used in optical lithography during device fabrication. Fabrication steps are schematically shown in Figure 1 starting from the Raman measurement of the bare graphene, where characteristic $2 \mathrm{D}$ and $\mathrm{G}$ peaks of graphene are visible along with the SiC peak. The reverse photolithography of the ohmic contacts are performed to form the source and drain contacts with a positive-tone photo-resist. After the development, $20 \mathrm{~nm}$ titanium and $80 \mathrm{~nm}$ gold are deposited by the electron beam evaporation, followed by a standard lift-off process. $100 \mu \mathrm{m} \times 100 \mu \mathrm{m}$ ohmic contacts are $120 \mu \mathrm{m}$ apart from each other, abbreviated as S and D in Fig. 1. After the ohmic contact metallization, mesa etch lithography is performed in order to isolate the devices electrically from each other. The sample 


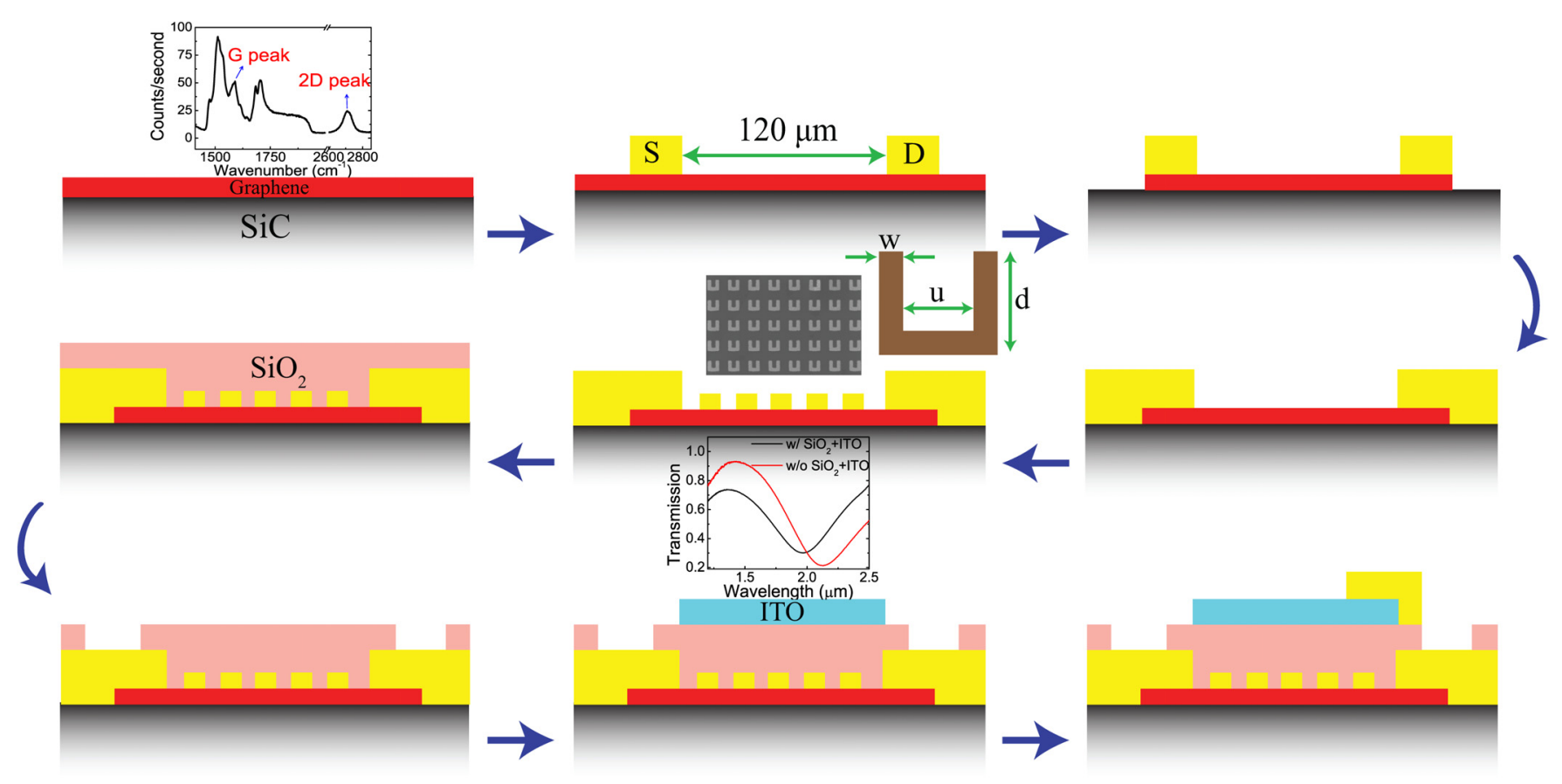

FIG. 1. Schematic of the device fabrication steps.

is exposed to oxygen plasma for $20 \mathrm{~s}$ by using Inductively Coupled Plasma Reactive Ion Etching (ICP-RIE), where the $\mathrm{RF}$ power is $50 \mathrm{~W}$ and ICP source power is $100 \mathrm{~W}$. Then, another photolithography for interconnect metallization, which consists of $20 \mathrm{~nm}$ titanium and $200 \mathrm{~nm}$ gold, is performed for the probing and wire bonding purposes during the measurements. This step provides large pads connected to source and drain contacts. Later, the fabrication of the split ring resonators is carried out by electron beam lithography on the active graphene region. $15 \mathrm{kV}$ accelerating voltage and $10 \mu \mathrm{m}$ aperture are used for the exposure. After electron beam lithography, $5 \mathrm{~nm}$ titanium as a buffer layer and $40 \mathrm{~nm}$ gold are evaporated. A scanning electron microscopy (SEM) image of an SRR array is shown as an inset of Fig. 1. Then, the dielectric passivation step is carried out with $100 \mathrm{~nm} \mathrm{SiO}_{2}$. This process is performed in two steps, where $50 \mathrm{~nm} \mathrm{SiO}_{2}$ is deposited by electron beam evaporation and $50 \mathrm{~nm} \mathrm{SiO}_{2}$ is deposited by sputter. Several trials showed that the gate leakage cannot be avoided by doing the electron beam evaporation step only. The reason is that the electron beam evaporation provides a very directional deposition on the surface, and it does not cover the sides of the metallic structures and the bumps due to the gold evaporation, and, hence, it leads to the leakage current caused by the SRRs, when the gate fabrication is completed. However, sputtering as a second step entirely covers all of the sides as well as the bumps on the metal surfaces such as a blanket, thus providing a proper isolation layer. The reason why electron beam evaporation is used before the sputtering is to protect the graphene surface from the plasma exposure of the sputter system. Argon plasma is created and used during the sputtering process. It was shown in Refs. 30 and 31 that the graphene is highly sensitive to Ar plasma, which can create defect sites on the surface. After the dielectric deposition steps, another etch process is performed in order to physically reach the source and drain contacts, as shown in the left bottom corner of Fig. $1 . \mathrm{CHF}_{3}$ plasma is used, where the RF power is set to $100 \mathrm{~W}$, and ICP power is set to $200 \mathrm{~W}$. Since the $\mathrm{SiC}$ substrate is semi-insulating, a top-gate is needed to apply the bias voltage. Indium tin oxide (ITO) is chosen to be the gate material due to its conductivity and transparency. Photolithography process is performed so that a $100 \mathrm{~nm}$-thick ITO window just above the active graphene region can be deposited by sputtering. As a final process step, a gold metal pad connected to the ITO is fabricated with the same steps followed in source-drain interconnect metallization.

IV measurements are performed in order to investigate the gate-dependent DC modulation performance of the devices. The results are plotted in Fig. 2. Fig. 2(a) shows the change of the IV characteristics between source and drain contacts under different gate voltages. Each curve is linear and hence has a constant resistance, which is the indication of the ohmic contact characteristics. Resistance increases up to $60 \mathrm{~V}$ of gate voltage and then decreases for higher gate voltages. This turning point can be seen clearly from the saddle point of Fig. 2(b). It can be concluded that the Dirac point, which is the charge neutrality point, is around $60 \mathrm{~V}$. Measurements are performed up to $70 \mathrm{~V}$ in order not to exceed the breakdown voltage of $\mathrm{SiO}_{2}$. Gate current at $\mathrm{V}_{\text {gate }}=60 \mathrm{~V}$ is measured to be on the orders of $10^{-11} \mathrm{~A}$, as shown in the inset of Figure 2(b), which proves that there is no gate leakage, and the dielectric passivation step works well.

Transmission of SRRs before and after the deposition of $\mathrm{SiO}_{2}$ and ITO layers is measured, and they are shown as an inset in Fig. 1. A red shift about $200 \mathrm{~nm}$ at the resonance wavelength is observed after the deposition of these two layers.

In this study, there are two resonant devices that operate at different wavelengths. Resonance wavelength of device 1 is at around $2 \mu \mathrm{m}$, where $\mathrm{u}=100 \mathrm{~nm}, \mathrm{w}=42 \mathrm{~nm}, \mathrm{~d}=180 \mathrm{~nm}$, and periodicity is $307 \mathrm{~nm}$ in both $\mathrm{x}$ and $\mathrm{y}$-directions. 

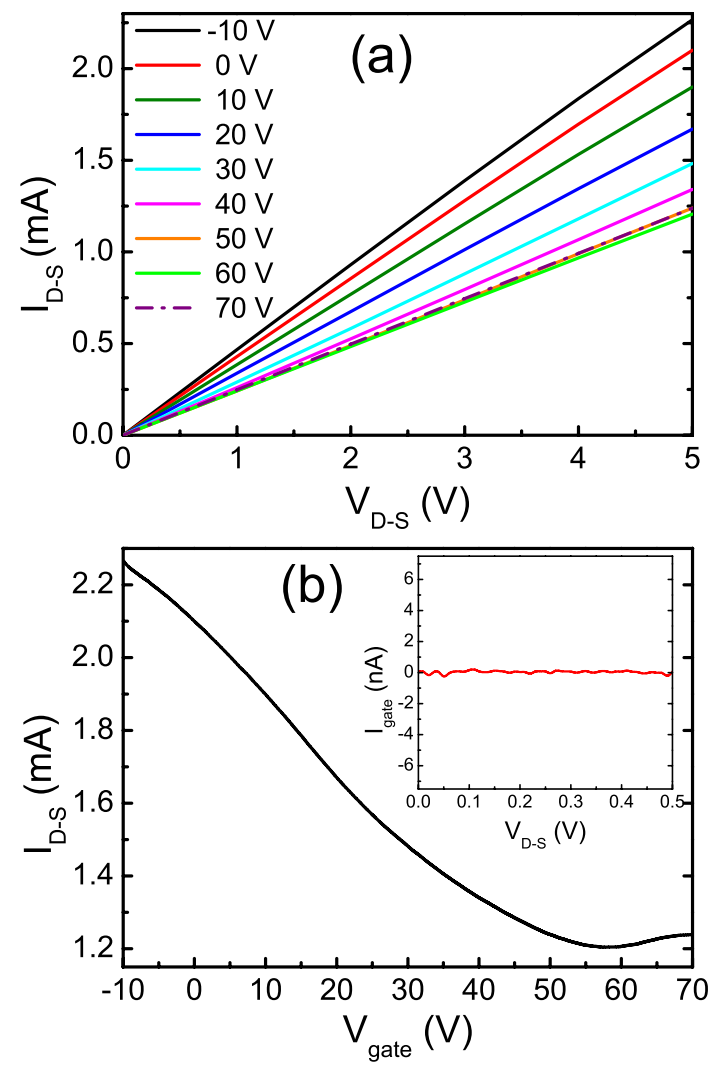

FIG. 2. DC-IV Measurements (a) drain voltage vs drain current, (b) gate voltage vs drain current; inset shows drain voltage vs gate current.

Resonance wavelength of device 2 is around $4 \mu \mathrm{m}$, where $\mathrm{u}=130 \mathrm{~nm}, \mathrm{w}=165 \mathrm{~nm}, \mathrm{~d}=420 \mathrm{~nm}$, and periodicity is $820 \mathrm{~nm}$ in both $\mathrm{x}$ and $\mathrm{y}$-directions. The transmission spectra of these two devices under different gate voltage differences, $\Delta V$, are shown in Fig. 3, where the resonance dips are enlarged in the inset. Here, $\Delta V$ is defined as $\Delta V=V_{\text {gate }}$ $-V_{C N P}$, where $V_{C N P}$ is the voltage value at the charge neutrality point. It should be noted that the curves change their shape asymmetrically with applied voltage. $28 \mathrm{~nm}$ blue shift at the resonance wavelength is measured and demonstrated in the inset of Fig. 3(b). The reason of this shift and the asymmetric behavior is the higher sensitivity of graphene to the light at higher wavelengths.

Line width and quality factor variations with respect to gate voltage difference are plotted in Fig. $4 . \Delta \lambda$ is the line width difference with respect to the line width of the curve measured at $\Delta V=0$. The line width decreases, and as a result the quality factor increases, as $\Delta \mathrm{V}$ decreases, which is to say that the curves narrow down away from the charge neutrality point. An incident photon having an energy $\hbar \omega>2 E_{F}$ can be absorbed because it generates an electron-hole pair. Fermi energy, $E_{F}$, approaches zero at the charge neutrality point, which results in more interband transitions due to the incident photons and hence absorption increases. The results presented in Figs. 3 and 4 are consistent with the theory. Transmission curve at the Dirac point, where $\Delta V=0 \mathrm{~V}$, is the broadest one having the lowest quality factor, as expected. The reason is that the absorption is the highest for this case.

Finite difference time domain (FDTD) simulations are carried out in order to verify experimentally observed
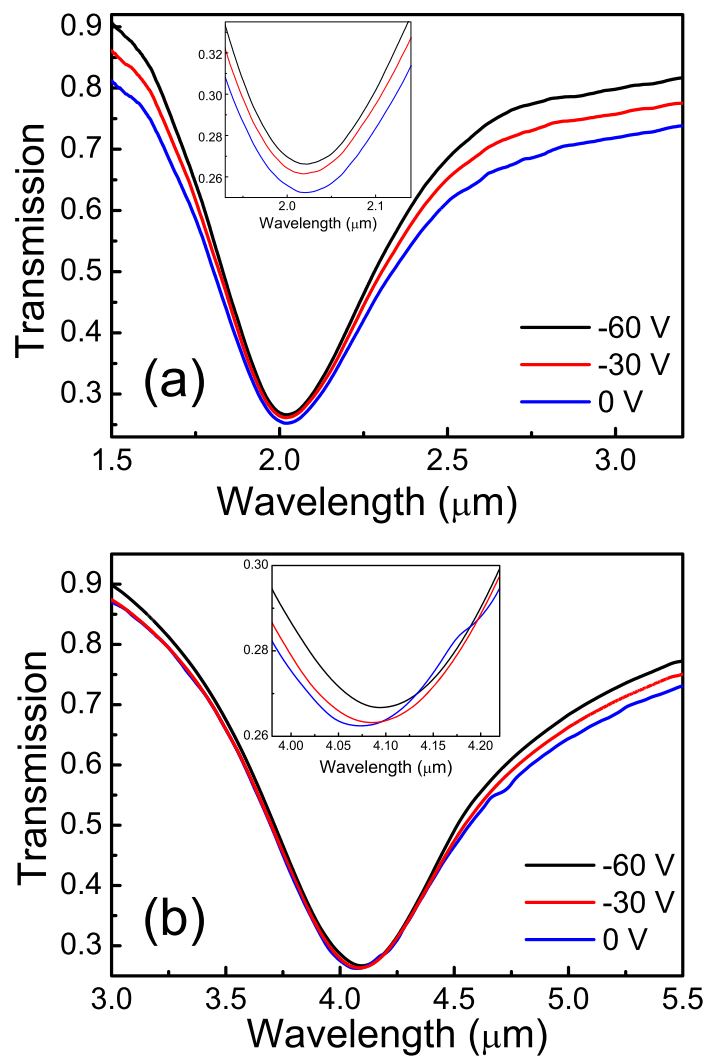

FIG. 3. Transmission measurements under different gate voltage differences, $\Delta V$, for (a) device 1 , and (b) device 2 .

transmission spectra. The results are shown in Fig. 5 for both device 1 and device 2. Graphene is modeled as an effective medium by using random phase approximation (RPA) model. ${ }^{25,26}$ Real and imaginary parts of refractive index are
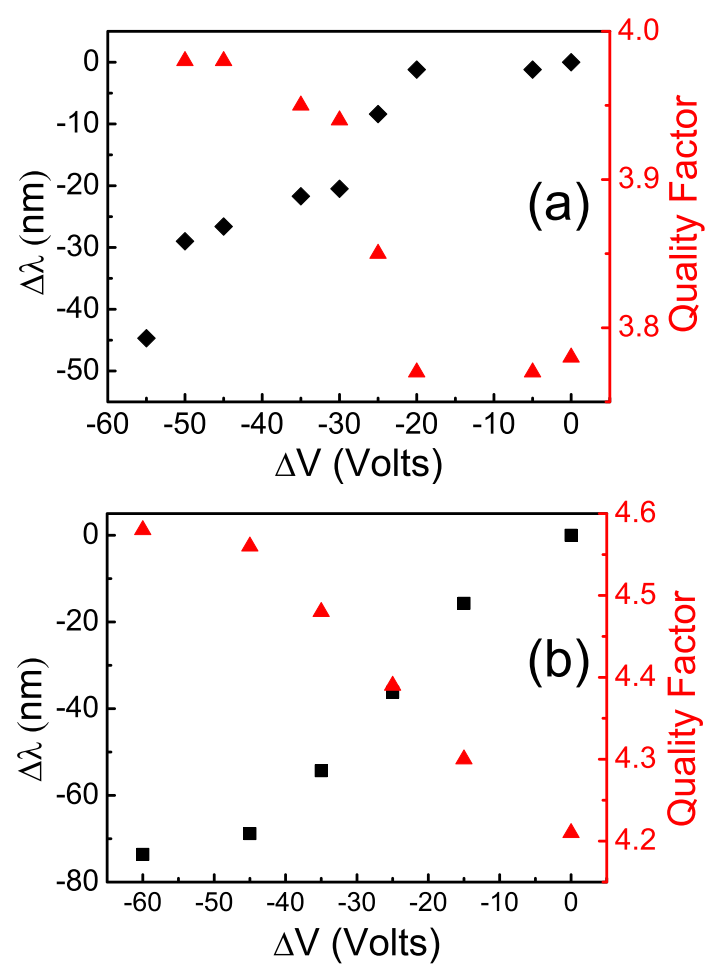

FIG. 4. Line width difference, $\Delta \lambda$, and quality factor variation for (a) device 1 and (b) device 2 (Black squares represent the line width difference, and red triangles represent the quality factor.). 

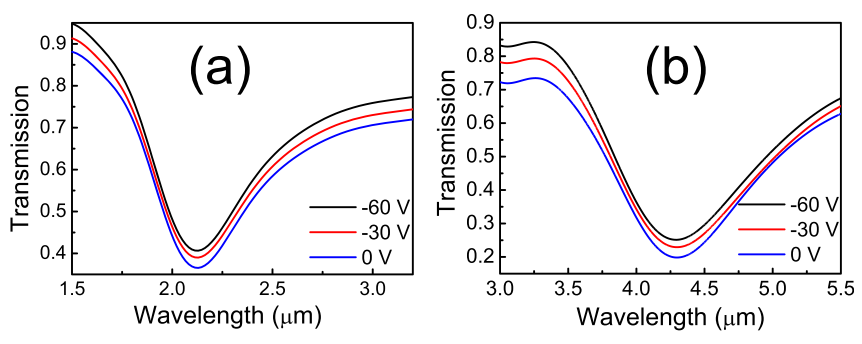

FIG. 5. Calculated transmission data under different gate voltage differences for (a) device 1 , and (b) device 2 .

extracted from the optical conductivity of graphene, which is a function of Fermi energy. The relation between the Fermi energy and the sheet carrier concentration of graphene is represented as $E_{F}=\hbar \nu_{F} \sqrt{\pi N}$. ${ }^{2,6}$ Furthermore, it is shown in Ref. 8 that the carrier concentration and the voltage are linearly proportional to each other. We used these two relations in our simulations in order to find out the corresponding Fermi energy for each applied voltage. Simulations show a good correlation with the measured data, where the differences may arise due to the fabrication imperfections, defect sites and grain boundaries on graphene. As the carrier concentration decreases, Fermi energy decreases, and more interband transitions are allowed resulting in a broader and damped resonance curve. In addition, the reason of the blue shift is that the real part of the refractive index changes at different electrical dopings.

In summary, we demonstrated resonance broadening and tuning of split ring resonators by utilizing an epitaxial graphene device with transparent top-gate. We obtained a frequency shift and electrical damping by using the graphene layer between the substrate and SRRs owing to the tunable carrier concentration of graphene, which consequently modulated its optical conductivity. Graphene based nanophotonics is an intense research due to a wide range of applications of this phenomenon. As SRRs are already being realized at optical frequencies, ${ }^{32,33}$ it is possible to use these SRRs and graphenebased structures for optical applications such as photovoltaics, ultrafast miniature photodetectors, and optical switches.

This work was supported by the projects DPT-HAMIT, ESF-EPIGRAT, NATO-SET-181, and TUBITAK under Project Nos. 107A004, 109A015, and 109E301. E.O. also acknowledges partial support from the Turkish Academy of Sciences. We also would like to thank Dr. Neval Cinel for the Raman measurement.

\footnotetext{
${ }^{1}$ A. K. Geim and K. S. Novoselov, Nat. Mater. 6, 183-191 (2007).

${ }^{2}$ K. S. Novoselov, A. K. Geim, S. V. Morozov, D. Jiang, M. I. Katsnelson, I. V. Grigorieva, S. V. Dubonos, and A. A. Firsov, Nature 438, 197-200 (2005).
}

${ }^{3}$ F. H. L. Koppens, D. E. Chang, and F. J. G. de Abajo, Nano Lett. 11, 3370-3377 (2011).

${ }^{4}$ K. I. Bolotin, K. J. Sikes, Z. Jiang, M. Klima, G. Fudenberg, J. Hone, P. Kim, and H. L. Stormer, Solid State Commun. 146, 351-355 (2008).

${ }^{5}$ K. S. Novoselov, A. K. Geim, S. V. Morozov, D. Jiang, Y. Zhang, S. V. Dubonos, I. V. Grigorieva, and A. A. Firsov, Science 306(5696), 666-669 (2004).

${ }^{6}$ Y. Zhang, Y. W. Tan, H. L. Stormer, and P. Kim, Nature 438, 201-204 (2005).

${ }^{7}$ X. Du, I. Skachko, A. Barker, and E. Y. Andrei, Nat. Nanotechnol. 3, 491-495 (2008).

${ }^{8}$ Z. Q. Li, E. A. Henriksen, Z. Jiang, Z. Hao, M. C. Martin, P. Kim, H. L. Stormer, and D. N. Basov, Nature Phys. 4, 532-535 (2008).

${ }^{9}$ F. Wang, Y. Zhang, C. Tian, C. Girit, A. Zettl, M. Crommie, and Y. R. Shen, Science 320, 206-209 (2008).

${ }^{10}$ F. Bonaccorso, Z. Sun, T. Hasan, and A. C. Ferrari, Nat. Photonics 4, 611-622 (2010).

${ }^{11}$ T. Hasan, Z. Sun, F. Wang, F. Bonaccorso, P. H. Tan, A. G. Rozhin, and A. C. Ferrari, Adv. Mater. 21, 3874-3899 (2009).

${ }^{12}$ Z. Sun, T. Hasan, F. Torrisi, D. Popa, G. Privitera, F. Wang, F. Bonaccorso, D. M. Basko, and A. C. Ferrari, ACS Nano 4, 803-810 (2010).

${ }^{13}$ R. J. Stoehr, R. Kolesov, J. Pflaum, and J. Wrachtrup, Phys. Rev. B 82, 121408(R) (2010).

${ }^{14}$ T. Gokus, R. R. Nair, A. Bonetti, M. Bhmler, A. Lombardo, K. S. Novoselov, A. K. Geim, A. C. Ferrari, A. A. Green, M. C. Hersam, and A. Hartschuh, in E-MRS Spring Meeting, 2010.

${ }^{15}$ T. Kampfrath, L. Perfetti, F. Schapper, C. Frischkorn, and M. Wolf, Phys. Rev. Lett. 95, 187403 (2005).

${ }^{16}$ K. F. Mak, J. Shan, and T. F. Heinz, Phys. Rev. Lett. 104, 176404 (2010).

${ }^{17}$ M. Breusing, C. Ropers, and T. Elsaesser, Phys. Rev. Lett. 102, 086809 (2009).

${ }^{18}$ X. S. Li, W. W. Cai, J. H. An, S. Kim, J. Nah, D. X. Yang, R. D. Piner, A. Velamakanni, I. Jung, E. Tutuc, S. K. Banerjee, L. Colombo, and R. S. Ruoff, Science 324, 1312-1314 (2009).

${ }^{19}$ V. V. Khardikov, E. O. Iarko, and S. L. Prosvirnin, J. Opt. 12, 045102 (2010).

${ }^{20}$ A. Bostwick, T. Ohta, T. Seyller, K. Horn, and E. Rotenberg, Nat. Phys. 3, 36-40 (2007).

${ }^{21}$ A. Bostwick, F. Speck, T. Seyller, K. Horn, M. Polini, R. Asgari, A. H. MacDonald, and E. Rotenberg, Science 328, 999-1002 (2010).

${ }^{22}$ J. Kim, H. Son, D. J. Cho, B. Geng, W. Regan, S. Shi, K. Kim, A. Zettl, Y. Shen, and F. Wang, Nano Lett. 12, 5598-5602 (2012).

${ }^{23}$ Y. Yao, M. A. Kats, P. Genevet, N. Yu, Y. Song, J. Kong, and F. Capasso, Nano Lett. 13, 1257-1264 (2013).

${ }^{24}$ N. K. Emani, T. F. Chung, X. Ni, A. V. Kildishev, Y. P. Chen, and A. Boltasseva, Nano Lett. 12, 5202-5206 (2012).

${ }^{25}$ L. A. Falkovsky and A. A. Varlamov, Eur. Phys. J. B 56, 281-284 (2007).

${ }^{26}$ L. A. Falkovsky and S. S. Pershoguba, Phys. Rev. B 76, 153410 (2007).

${ }^{27}$ Y. Liu, R. F. Willis, K. V. Emtsev, and T. Seyller, Phys. Rev. B 78, 201403 (2008).

${ }^{28}$ C. Tegenkamp, H. Pfnur, T. Langer, J. Baringhaus, and H. W. Schumacher, J. Phys.: Condens. Matter 23, 012001 (2011).

${ }^{29}$ V. W. Brar, S. Wickenburg, M. Panlasigui, C. Park, T. O. Wehling, Y. Zhang, R. Decker, C. Girit, A. V. Balatsky, S. G. Louie, A. Zettl, and M. F. Crommie, Phys. Rev. Lett. 104, 036805 (2010).

${ }^{30}$ M. M. Ugeda, I. Brihuega, F. Guinea, and J. M. Gomez-Rodriguez, Phys. Rev. Lett. 104, 096804 (2010).

${ }^{31}$ K. Kim, H. Kang, C. Y. Lee, and W. S. Yun, J. Vac. Sci. Technol. B 31, 030602 (2013).

${ }^{32}$ K. Aydin, A. O. Cakmak, L. Sahin, Z. Li, F. Bilotti, L. Vegni, and E. Ozbay, Phys. Rev. Lett. 102, 013904 (2009).

${ }^{33}$ L. Sahin, K. Aydin, G. Turhan-Sayan, and E. Ozbay, J. Nanophotonics 5, 051812 (2011). 